\title{
Challenges and Applications of Automated Extraction of Socio-political Events from Text (CASE 2021): Workshop and Shared Task Report
}

\author{
Ali Hürriyetoğlu \\ Koç University \\ Sarıyer, İstanbul, Turkey \\ ahurriyetogluaku.edu.tr
}

\author{
Jakub Piskorski \\ Polish Academy of Sciences \\ Warsaw, Poland \\ jpiskorski@gmail.com
}

\author{
Osman Mutlu \\ Koç University \\ Sarıyer, İstanbul, Turkey \\ omutlu@ku.edu.tr
}

\author{
Hristo Tanev \\ European Commission \\ Ispra, Varese, Italy \\ hristo.tanev@ec.europa.eu \\ Reyyan Yeniterzi \\ Sabanc1 University \\ Tuzla, İstanbul, Turkey \\ reyyanesabanciuniv.edu
}

\author{
Deniz Yüret \\ Koç University \\ Sariyer, İstanbul, Turkey \\ dyureteku.edu.tr
}

Vanni Zavarella

European Commission

Ispra, Varese, Italy

vanni.zavarelladec.europa.eu

\author{
Erdem Yörük \\ Koç University \\ Sarıyer, İstanbul, Turkey \\ eryorukeku.edu.tr
}

\author{
Aline Villavicencio \\ The University of Sheffield \\ Sheffield, United Kingdom \\ a.villavicenciodsheffield.ac.uk
}

\section{Abstract}

This workshop is the fourth issue of a series of workshops on automatic extraction of sociopolitical events from news, organized by the Emerging Market Welfare Project, with the support of the Joint Research Centre of the European Commission and with contributions from many other prominent scholars in this field. The purpose of this series of workshops is to foster research and development of reliable, valid, robust, and practical solutions for automatically detecting descriptions of sociopolitical events, such as protests, riots, wars and armed conflicts, in text streams. This year workshop contributors make use of the stateof-the-art NLP technologies, such as Deep Learning, Word Embeddings and Transformers and cover a wide range of topics from text classification to news bias detection. Around 40 teams have registered and 15 teams contributed to three tasks that are i) multilingual protest news detection, ii) fine-grained classification of socio-political events, and iii) discovering Black Lives Matter protest events. The workshop also highlights two keynote and four invited talks about various aspects of creating event data sets and multi- and cross-lingual machine learning in few- and zero-shot settings.

\section{Introduction}

Today, the unprecedented quantity of easily accessible data on social, political, and economic processes offers ground-breaking potential in guiding data-driven analysis in social and human sciences and in influencing policy-making processes. The need for precise and high-quality information about a wide variety of events ranging from political violence, environmental catastrophes, and conflict to international economic and health crises has rapidly escalated (Della Porta and Diani, 2015; Coleman et al., 2014). Governments, multilateral organizations, and local and global NGOs present an increasing demand for this data to prevent or resolve conflicts, provide relief for those that are afflicted, or improve the lives of and protect citizens in a variety of ways. For instance, Black Lives Matter protests ${ }^{1}$, conflict in Syria ${ }^{2}$ and COVID-19 related events ${ }^{3}$ are only a few examples where we must understand, analyze, and improve the real-life situations using such data.

A recent report from ReliefWeb ${ }^{4}$ clearly demon-

\footnotetext{
${ }^{1}$ http: / /protestmap.raceandpolicing.com, accessed on June 2, 2021.

${ }^{2}$ https: //www. cartercenter. org/peace/conflict_resolution/ syria-conflict-resolution.html, accessed on June 2, 2021.

${ }^{3}$ https://en.wikipedia.org/wiki/ Protests_over_responses_to_the_COVID-19_ pandemic, accessed on June 2, 2021.

${ }^{4}$ https://reliefweb.int/report/world/ trends-armed-conflict-1946-2017, accessed on June 3, 2021.
} 
strates that the number of wars and other armed conflicts is on an increasing trend. In particular, the so-called internationalized conflicts are on a rise in the last two decades. In this situation, it is important to provide solutions for situation awareness, using various branches of artificial intelligence (AI), natural language processing (NLP), machine learning (ML), and advanced statistical methods.

In this clue, event detection and extraction plays an important role, because of its capacity to detect conflict developments in news and social media and to extract important information about them. Such information involves the quantity and the profiles of the victims, the participating entities, the conflict dynamics, its spatio-temporal characteristics, the weaponry used, as well as infrastructural, technical and human impact. This information extracted through various NLP methods can throw light on the intensity and the trend development of each conflict, as it is reflected in the media. Event detection has been used by political analysts to write their daily situation reports for decision makers, to create long-term analyses, as well as for conflict forecasting and prediction.

Automation offers scholars not only the opportunity to improve existing practices, but also to vastly expand the scope of data that can be collected and studied, thus potentially opening up new research frontiers within the field of socio-political events, such as political violence and social movements. Event information collection has long been a challenge for the NLP community as it requires sophisticated methods in defining event ontologies, creating language resources, and developing algorithmic approaches (Pustejovsky et al., 2003; Tanev et al., 2008; Emanuela, 2018; Chen et al., 2021). We believe that this workshop and the shared task contribute strongly towards putting emphasis on this important technology, providing a gathering point for scientists and developers in NLP, AI, conflict studies and related areas.

Social and political scientists have been creating event databases such as ACLED (Raleigh et al., 2010), EMBERS (Saraf and Ramakrishnan, 2016), GDELT (Leetaru and Schrodt, 2013), ICEWS (O'Brien, 2010), MMAD (Weidmann and Rød, 2019), PHOENIX, POLDEM (Kriesi et al., 2019), SPEED (Nardulli et al., 2015), TERRIER (Liang et al., 2018), and UCDP (Sundberg et al., 2012) for decades. These projects and the new ones increasingly rely on machine learn- ing (ML) and NLP methods to deal better with the vast amount and variety of data in this domain (Hürriyetoğlu et al., 2021). Nonetheless, automated approaches suffer from major issues like bias, low generalizability, class imbalance, training data limitations, ethical issues, and lack of recall quantification which affect the quality of the results and their use drastically (Leins et al., 2020; Bhatia et al., 2020; Chang et al., 2019; Yörük et al., 2021). Moreover, the results of the automated systems for socio-political event information collection may not be comparable to each other or not of sufficient quality (Wang et al., 2016; Schrodt, 2020).

Socio-political events are varied and nuanced. Both the political context and the local language used may affect whether and how they are reported. Therefore, all steps of information collection (event definition, language resources, and manual or algorithmic steps) may need to be constantly updated. This leads us to a series of challenging questions such as: Do events related to minority groups are represented well? Are new types of events covered? Are the event definitions and their operationalization comparable across systems? We organize the workshop on Challenges and Applications of Automated Extraction of Socio-political Events from Text (CASE 2021) ${ }^{5}$ and the shared task Sociopolitical and Crisis Events Detection ${ }^{6}$ to seek answers to these and related questions, to inspire innovative technological and scientific solutions for tackling the aforementioned issues, and to quantify the quality of the automated event extraction systems. Moreover, the workshop aims to trigger a deeper understanding of the performance of the computational tools used and the usability of the resulting socio-political event datasets. The workshop is co-located with the Joint Conference of the 59th Annual Meeting of the Association for Computational Linguistics and the 11th International Joint Conference on Natural Language Processing (ACL-IJCNLP 2021).

We invited contributions from researchers in computer science, NLP, ML, AI, socio-political sciences, conflict analysis and forecasting, peace studies, as well as computational social science scholars involved in the collection and utilization of socio-political event data. Social and political scientists are interested in reporting and discussing

\footnotetext{
${ }^{5}$ https://emw.ku.edu.tr/case-2021/, accessed on June 9, 2021.

${ }^{6}$ https://github.com/emerging-welfare/ case-2021-shared-task, June 12, 2021.
} 
their approaches and observe what the state-of-theart text processing systems can achieve for their domain. Computational scholars have the opportunity to illustrate the capacity of their approaches in this domain and benefit from being challenged by real-world use cases. Academic workshops specific to tackling event information in general or for analyzing text in specific domains such as health, law, finance, and biomedical sciences have significantly accelerated progress in these topics and fields, respectively. However, there is not a comparable effort for handling socio-political events. We hope to fill this gap and contribute to social and political sciences in a similar spirit. We invite work on all aspects of automated coding of sociopolitical events from mono- or multi-lingual text sources. This includes (but is not limited to) the broad topics below.

Data: collecting and annotating data, identifying the qualities, bias and fairness of the sources, handling ethics, misinformation, privacy, and fairness concerns pertaining to event datasets, respecting copyright of the sources at the creation, dissemination, and release phases of an event dataset;

Task: defining, populating, and facilitating event schemas and ontologies, extracting events in and beyond a sentence, detecting event coreference and event-event relations such as subevents, main events, and causal relations, investigating lexical, syntactic, and pragmatic aspects of event information manifestation, determining socio-poltical events pertaining to societal issues such as COVID-19 and BLM, detecting novel events;

Approaches: developing rule-based, machine learning, hybrid, and human-in-the-loop approaches for creating event datasets; and

Evaluation: evaluating event datasets in light of reliability and validity metrics, estimating what is missing in event datasets using internal and external information, utilizing event datasets, releasing of new event datasets.

We provide summaries of the accepted papers, the shared task, keynotes, and invited talks in the sections 2, 3, 4, and 5 respectively. Section 6 concludes this report with main lessons derived from these efforts and interactions.

\section{Accepted Papers}

The workshop attracted 21 submissions. The competition was high and 7 of them were accepted based on reviewer evaluations, which vary between 4 and 6 for each paper.

Here are brief descriptions of all accepted papers, except from the ones participating in the shared task, which are described in other papers in this proceedings:

de Vroe et al. (2021) present an open domain, lexicon-based event extraction system that captures various types of event modality. The definition of "event" in this work is quite broad, i.e. every predicate construction is taken into consideration. The authors use syntactic parsing to detect the event modality, which is a very important phenomena when making distinction between current, past and just probable events. The system explores conditionality, counterfactuality, negation, and propositional attitude. The achieved accuracy in the modality labelling task is $0.81 \mathrm{~F} 1$ that is measured on a small corpus of 100 manually annotated predicates.

Raza (2021) explores the topic of detecting fake news, which is potentially related to the trustability of the sources, from which events are extracted. The main approach in this study is based on a modified version of a pre-trained Bidirectional Encoder Representations from Transformers (BERT) with the capability to receive as input news-related and side information. In particular, each news item is represented by its title (main information) and side information, such as temporal, news-related information, author and source, as well as social contexts (related tweets) which give information about users' reactions on the news. The proposed model is quite promising, considering it outperforms all other state-of-the-art methods. It achieves 96\% accuracy in deciding between fake and real news on a test set with fake and real news nearly equally represented.

Caselli et al. (2021) explore how efficiently a retrained BERT model detects protest events. Authors present the PROTEST-ER system, which uses a retrained BERT model for protest event extraction. They use annotated event data from the protest event detection task following the 2019 CLEF ProtestNews Lab (Hürriyetoğlu et al., 2019a,b). A worth-to-mention finding of this work is that PROTEST-ER outperforms a corresponding generic BERT with 8.1 points.

Ramrakhiyani et al. (2021) describe a deep learn- 
ing approach for detecting incidents from industrial reports. Incidents in industries have huge social and political impacts. However, automated analysis of repositories of incident reports has remained a challenge. Due to absence of event annotated datasets for industrial incidents authors employ a transfer learning based approach. A detailed analysis is provided on how amount of data utilized affect pre-training and why pre-training improves the performance. Data is gathered from aviation and construction incident reports. Different deep learning methods are evaluated for the task, including BiLSTM and transfer learning. Transfer learning consistently outperforms the baseline and achieves F1 measure of 0.81 .

Radford (2021) presents a study on geocoding and a new data set. Geocoding is an important subtask of event detection, in which the goal is to find the geographic coordinates associated with event descriptions. The paper presents an "end-to-end probabilistic model" for geocoding from text data. A novel data set has been created for evaluating the performance of geocoding systems. The output of the new model is compared with a state-of-the-art model, called Mordecai. The comparison clearly shows an improvement provided by the proposed model.

Scharf et al. (2021) report on a study on the political bias in Hong Kong published news reporting about protest events. The paper reports on lexical differences between home and Western news sources about protests happening in Hong Kong in the period 1998-2020. Experiments on topic modeling, sentiment analysis, lexical distribution and comparative lexical analysis between Westernand Hong Kong-based sources reveal a bias in the reporting from the Hong Kong press. The evaluation reveals that during the Anti-Extradition Law Amendment Bill Movement reports from Hong Kong made fewer references to police violence compared to the Western media. The study also reveals that the lexical contexts of salient keywords changed in Hong Kong sources when the Movement emerged.

Kar et al. (2021) describe an algorithm for event argument detection and aggregation. The paper reports on document level aggregation of the following argument types: Time, Place, Casualties, After-Effect, Reason, and Participant. The ArgFuse algorithm is based on a BERT based active learning classifier, which identifies whether a pair of event arguments is redundant, and a Biased Text Rank argument ordering function. Authors report F1 measure of 0.61 , which beats all the other 5 baseline algorithms with which the ArgFuse performance is compared.

\section{Shared Task: Socio-political and Crisis Events Detection}

The work on event database creation comprises of three steps that are collecting events, classifying them, and measuring utility of the system output, which is an event database, against ground-truth. Each of these steps contains pitfalls and subject to limitations. For instance, the data source utilized maybe biased or a ground-truth may not be available. Although aforementioned issues in sociopolitical and crisis event studies have been studied by numerous scholars for decades to date, there are still no answers or solutions to them (Wang et al., 2016; Lorenzini et al., 2016; Schrodt, 2020; Raleigh, 2020; Eck, 2021; Boschee, 2021). Therefore, we aim at contributing to the understanding and resolution of event database creation via quantifying performance of the state-of-the-art text processing systems in the shared task Socio-political and Crisis Events Detection. ${ }^{7}$

The shared task consists of three tasks that are on collection (Task 1), classification (Task 2) (Haneczok et al., 2021), and evaluation (Task 3) of event databases. Shared task and submission details are reported in the overview papers of the tasks (Hürriyetoğlu et al., 2021; Haneczok et al., 2021; Giorgi et al., 2021) and the system description papers in this proceedings respectively. We provide a summary of the tasks and the findings in the following subsections.

\subsection{Task 1: Multilingual protest news detection}

The task is designed to be both multilingual (having both training and test data in English, Portuguese, and Spanish) and cross-lingual (having data in Hindi only for test). There are four subtasks that are document classification (subtask 1), sentence classification (subtask 2), event sentence classification (subtask 3), and event extraction (subtask 4). Event information is at the center of all of the subtasks, i.e. documents and sentences are classified as containing event information in subtasks

\footnotetext{
https://github.com/emerging-welfare/ case-2021-shared-task, accessed on June 9, 2021.
} 
1 and 2, sentences that are about the same event are identified in subtask 3 , and event trigger and its arguments are extracted in subtask 4.

13 teams have submitted 238 submissions for the evaluation scenarios specified with subtask and language combinations. The best submissions utilized deep learning approaches that combine the training data in various languages, utilize large models, further re-train the models, and create ensemble models (Awasthy et al., 2021; Hettiarachchi et al., 2021; Re et al., 2021; Hu and Stoehr, 2021; Tan et al., 2021). Although training data was limited in Portuguese and Spanish and not available in Hindi, the best performing participants managed to deliver predictions that are between 77.27 and $93.03 \mathrm{~F} 1-$ macro in subtasks 1, 2, and 3 for all languages. The performance of the best system for subtask 4 for all languages was between 66.20 and 78.11 for all languages and 4-5 F1-macro points ahead of all other teams in all languages.

\subsection{Task 2: Fine-grained Event Classification in News-like Text Snippets}

Task 2 aims at evaluating conventional and generalized zero-shot learning event classification approaches to classify short text snippets reporting socio-political and crisis events. The task is divided into three subtasks: (a) classification of text snippets reporting socio-political events, using 25 events classes from the Armed Conflict Location and Event Data Project (ACLED) event taxonomy(Raleigh et al., 2010), for which vast amount of training data exists, although exhibiting slightly different structure and style vis-a-vis test data, (b) enhancement to a generalized zero-shot learning problem, where 3 additional event types were introduced in advance, but without any training data ('unseen' classes), and (c) further extension, which introduced 2 additional event types, announced shortly prior to the evaluation phase. Task 2 focuses on classification of events in English texts and the event definitions of events in this task are not fully compatible with those in Task 1 .

8 teams registered, out of which 4 returned system responses, for Task 2. Best performing systems for the subtask 1, 2 and 3 achieved 83.9\%, 79.7\% and $77.1 \%$ weighted $F_{1}$ scores respectively. Most of the solutions submitted are built on top of finetuned Transformer-based models like BERT and ROBERTA. Given the specific set up of this task, i.e., the training data being some-what different from the test data and inclusion of some unseen classes the top results obtained can be considered good, however, there is place for improvement.

\subsection{Task 3: Discovering Black Lives Matter events in United States}

Task 3 is only an evaluation task where the participants of Task 1 have the possibility to evaluate their systems on reproducing a manually curated Black Lives Matter (BLM) related protest event list. Participants use document collections, provided by the organizers and different from the documents from where Gold Standard has been extracted, to extract place and date of the BLM events in these collections. The event definition applied for determining these events is the same as the one facilitated for task 1. Participants may utilize any other data source to improve performance of their submissions. The goal of the task is to achieve as high correlation as possible with the events from the Gold Standard, as computed by aggregating events on a regular cell geographical grid..

5 teams that performed the best in Task 1 were invited to participate in this task. In general all participating systems showed low levels of correlation with the Gold standard data, including the baseline system. The low recall at this year's shared task is most probably due to the low coverage of the test corpus, which participating systems have used, and its poor overlapping with manually collected Gold Standard Data. Two systems showed a relatively good performance: NoConflict $\mathrm{Hu}$ and Stoehr (2021) and EventMiner Hettiarachchi et al. (2021). The main lesson from this task is that Gold Standard data and test data should be checked for consistency and correlation. Moreover, this evaluation task highlighted some of the current limits on the usability of automatically extracted event datasets for modelling socio-political processes, such as fine-grained geocoding of events.

\section{Keynotes}

Kristine Eck and Elizabeth Boschee will deliver the keynote talks. Eck (2021) addresses the responsibility of the scholars that create event datasets to define and apply what is right, suggests data sources alternative to news data that may report event information inconsistently, and emphasizes the need for interdisciplinary collaboration for creating data sets that advance conflict studies. Boschee et al. (2013); Boschee (2021) share the concerns addressed by 
Prof. Eck and presents a detailed study that compares various approaches for utilizing multilingual data in a cross-lingual zero-shot setting to improve quality of the event datasets.

\section{Invited Talks}

The workshop contains an invited talks session as well. The authors of the papers published in Findings of ACL and related to workshop theme are invited to present their work in this session. The papers are

Zhou et al. (2021) propose an event-driven trading strategy that predicts stock movements by detecting corporate events from news articles;

Halterman et al. (2021) introduce the IndiaPoliceEvents Corpus-all 21,391 sentences from 1,257 Times of India articles about events in the state of Gujarat during March 2002;

Halterman and Radford (2021) show utility of "upsampling" coarse document labels to finegrained labels or spans for protest size detection; and

Tsarapatsanis and Aletras (2021) discuss the importance of academic freedom, the diversity of legal and ethical norms, and the threat of moralism in the computational law field.

\section{Conclusion}

This workshop is the fourth event from a series of workshops on automatic extraction of sociopolitical events from news, organized by the Emerging Market Welfare Project, with the support of the Joint Research Centre of the European Commission, with contributions from many other prominent scholars in this field. The purpose of this series of workshops is to foster research and development in the area of event extraction of socio-political events.

The topics cover a wide range of applications and technologies: event detection via text classification, detection of news bias, fake news detection, modality analysis through syntactic parsing, event argument extraction and aggregation, a new geocoding algorithm and the creation of a new geocoding dataset. Most of the papers are dedicated to protest events, one paper is about industrial reports, and one paper discusses generic events, not related to the socio-political topic.
The papers in this issue of the workshop make use of state-of-the-art NLP technologies, such as Deep Learning, Word Embeddings and Transformers. Most of the papers use the BERT model: some use the pre-trained existing models, others train domain-specific ones, and one of the paper introduces a modified version of BERT. Most papers use BERT embeddings as features in their models and one paper discusses an algorithm, which uses a full syntactic parser. Sentiment analysis is used in one paper, which studies the political bias of the news.

The shared task results shed light on critical aspects of the automated socio-political extraction and evaluation methodology. ${ }^{8}$

\section{Acknowledgments}

The authors from Koc University were funded by the European Research Council (ERC) Starting Grant 714868 awarded to Dr. Erdem Yörük for his project Emerging Welfare.

\section{References}

Parul Awasthy, Jian Ni, Ken Barker, and Radu Florian. 2021. IBM MNLP IE at CASE 2021 task 1: Multi-granular and multilingual event detection on protest news. In Proceedings of the 4th Workshop on Challenges and Applications of Automated Extraction of Socio-political Events from Text (CASE 2021), online. Association for Computational Linguistics (ACL).

Shraey Bhatia, Jey Han Lau, and Timothy Baldwin. 2020. You are right. i am alarmed-but by climate change counter movement. arXiv preprint arXiv:2004.14907.

Elizabeth Boschee. 2021. Keynote Abstract: Events on a Global Scale: Towards Language-Agnostic Event Extraction. In Proceedings of the 4th Workshop on Challenges and Applications of Automated Extraction of Socio-political Events from Text (CASE 2021), online. Association for Computational Linguistics (ACL).

Elizabeth Boschee, Premkumar Natarajan, and Ralph Weischedel. 2013. Automatic Extraction of Events from Open Source Text for Predictive Forecasting. In V.S. Subrahmanian, editor, Handbook of Computational Approaches to Counterterrorism, pages 5167. Springer New York, New York, NY.

\footnotetext{
${ }^{8}$ Detailed results are summarized in the overview papers (Hürriyetoğlu et al., 2021; Haneczok et al., 2021), and evaluation (Task 3) (Giorgi et al., 2021) of event databases. Moreover, system description papers provide full details
} 
Tommaso Caselli, Osman Mutlu, Angelo Basile, and Ali Hürriyetoğlu. 2021. PROTEST-ER: Retraining BERT for Protest Event Extraction. In Proceedings of the 4th Workshop on Challenges and Applications of Automated Extraction of Socio-political Events from Text (CASE 2021), online. Association for Computational Linguistics (ACL).

Kai-Wei Chang, Vinod Prabhakaran, and Vicente Ordonez. 2019. Bias and fairness in natural language processing. In Proceedings of the 2019 Conference on Empirical Methods in Natural Language Processing and the 9th International Joint Conference on Natural Language Processing (EMNLP-IJCNLP): Tutorial Abstracts, Hong Kong, China. Association for Computational Linguistics.

Muhao Chen, Hongming Zhang, Qiang Ning, Manling Li, Heng Ji, Kathleen McKeown, and Dan Roth. 2021. Event-centric natural language understanding.

Peter T Coleman, Morton Deutsch, and Eric C Marcus. 2014. The handbook of conflict resolution: Theory and practice. John Wiley \& Sons.

Donatella Della Porta and Mario Diani. 2015. The Oxford handbook of social movements. Oxford University Press.

Kristine Eck. 2021. Keynote Abstract: Machine Learning in Conflict Studies: Reflections on Ethics, Collaboration, and Ongoing Challenges. In Proceedings of the 4th Workshop on Challenges and Applications of Automated Extraction of Socio-political Events from Text (CASE 2021), online. Association for Computational Linguistics (ACL).

Boroş Emanuela. 2018. Neural Methods for Event Extraction. Ph.D. thesis, Université Paris-Saclay.

Salvatore Giorgi, Vanni Zavarella, Hristo Tanev, Nicolas Stefanovitch, Sy Hwang, Hansi Hettiarachchi, Tharindu Ranasinghe, Vivek Kalyan, Paul Tan, Shaun Tan, Martin Andrews, Hu Tiancheng, Niklas Stoehr, Francesco Ignazio Re, Daniel Vegh, Dennis Atzenhofer, Brenda Curtis, and Ali Hürriyetoğlu. 2021. Discovering Black Lives Matter events in the United States - Shared Task 3, CASE 2021. In Proceedings of the 4th Workshop on Challenges and Applications of Automated Extraction of Sociopolitical Events from Text (CASE 2021), online. Association for Computational Linguistics (ACL).

Andrew Halterman, Katherine Keith, Sheikhand Sarwar, and Brendan O'Connor. 2021. Corpus-level evaluation for event qa: The indiapoliceevents corpus covering the 2002. In Proceedings of the 59th Annual Meeting of the Association for Computational Linguistics, Online. Association for Computational Linguistics.

Andrew Halterman and Benjamin J. Radford. 2021. Few-shot upsampling for protest size detection. In
Proceedings of the 59th Annual Meeting of the Association for Computational Linguistics, Online. Association for Computational Linguistics.

Jacek Haneczok, Guillaume Jacquet, Jakub Piskorski, and Nicolas Stefanovitch. 2021. Fine-grained event classification in news-like text snippets shared task 2, CASE 2021. In Proceedings of the 4th Workshop on Challenges and Applications of Automated Extraction of Socio-political Events from Text (CASE 2021), online. Association for Computational Linguistics (ACL).

Hansi Hettiarachchi, Mariam Adedoyin-Olowe, Jagdev Bhogal, and Mohamed Gaber. 2021. DAAI at CASE 2021 Task 1: Transformer-based Multilingual Sociopolitical and Crisis Event Detection. In Proceedings of the 4th Workshop on Challenges and Applications of Automated Extraction of Socio-political Events from Text (CASE 2021), online. Association for Computational Linguistics (ACL).

Tiancheng Hu and Niklas Stoehr. 2021. Team "NoConflict" at CASE 2021 Task 1:Pretraining for SentenceLevel Protest Event Detection. In Proceedings of the 4th Workshop on Challenges and Applications of Automated Extraction of Socio-political Events from Text (CASE 2021), online. Association for Computational Linguistics (ACL).

Ali Hürriyetoğlu, Osman Mutlu, Farhana Ferdousi Liza, Erdem Yörük, Ritesh Kumar, and Shyam Ratan. 2021. Multilingual protest news detection - Shared Task 1, CASE 2021. In Proceedings of the 4th Workshop on Challenges and Applications of Automated Extraction of Socio-political Events from Text (CASE 2021), online. Association for Computational Linguistics (ACL).

Ali Hürriyetoğlu, Erdem Yörük, Deniz Yüret, Çağrı Yoltar, Burak Gürel, Fırat Duruşan, and Osman Mutlu. 2019a. A task set proposal for automatic protest information collection across multiple countries. In Advances in Information Retrieval, pages 316-323, Cham. Springer International Publishing.

Ali Hürriyetoğlu, Erdem Yörük, Deniz Yüret, Çağrı Yoltar, Burak Gürel, Firat Duruşan, Osman Mutlu, and Arda Akdemir. 2019b. Overview of CLEF 2019 Lab ProtestNews: Extracting Protests from News in a Cross-Context Setting. In Experimental IR Meets Multilinguality, Multimodality, and Interaction, pages 425-432, Cham. Springer International Publishing.

Ali Hürriyetoğlu, Erdem Yörük, Osman Mutlu, Firat Duruşşan, Çağrı Yoltar, Deniz Yüret, and Burak Gürel. 2021. Cross-Context News Corpus for Protest Event-Related Knowledge Base Construction. Data Intelligence, 3(2):308-335.

Debanjana Kar, Sudeshna Sarkar, and Pawan Goyal. 2021. ArgFuse: A Weakly-Supervised Framework for Document-Level Event Argument Aggregation. In Proceedings of the 4th Workshop on Challenges 
and Applications of Automated Extraction of Sociopolitical Events from Text (CASE 2021), online. Association for Computational Linguistics (ACL).

Hanspeter Kriesi, Bruno Wüest, Jasmine Lorenzini, Peter Makarov, Matthias Enggist, Klaus Rothenhäusler, Thomas Kurer, Silja Häusermann, and Altiparmakis Patrice Wangen. 2019. Poldem-protest event dataset 30 .

Kalev Leetaru and Philip A Schrodt. 2013. GDELT: Global data on events, location, and tone, 19792012. In ISA annual convention, volume 2, pages 1-49. Citeseer.

Kobi Leins, Jey Han Lau, and Timothy Baldwin. 2020. Give me convenience and give her death: Who should decide what uses of NLP are appropriate, and on what basis? In Proceedings of the 58th Annual Meeting of the Association for Computational Linguistics, pages 2908-2913, Online. Association for Computational Linguistics.

Yan Liang, Khaled Jabr, Christan Grant, Jill Irvine, and Andrew Halterman. 2018. New techniques for coding political events across languages. In 2018 IEEE International Conference on Information Reuse and Integration (IRI), pages 88-93.

Jasmine Lorenzini, Peter Makarov, Hanspeter Kriesi, and Bruno Wueest. 2016. Towards a Dataset of Automatically Coded Protest Events from Englishlanguage Newswire Documents. In Paper presented at the Amsterdam Text Analysis Conference.

Peter F. Nardulli, Scott L. Althaus, and Matthew Hayes. 2015. A Progressive Supervised-learning Approach to Generating Rich Civil Strife Data. Sociological Methodology, 45(1):148-183.

Sean P. O’Brien. 2010. Crisis Early Warning and Decision Support: Contemporary Approaches and Thoughts on Future Research. International Studies Review, 12(1):87-104.

James Pustejovsky, José M Castano, Robert Ingria, Roser Sauri, Robert J Gaizauskas, Andrea Setzer, Graham Katz, and Dragomir R Radev. 2003. Timeml: Robust specification of event and temporal expressions in text. New directions in question answering, 3:28-34.

Benjamin J. Radford. 2021. Regressing Location on Text for Probabilistic Geocoding. In Proceedings of the 4th Workshop on Challenges and Applications of Automated Extraction of Socio-political Events from Text (CASE 2021), online. Association for Computational Linguistics (ACL).

Clionadh Raleigh. 2020. Keynote abstract: Too soon? the limitations of AI for event data. In Proceedings of the Workshop on Automated Extraction of Sociopolitical Events from News 2020, page 7, Marseille, France. European Language Resources Association (ELRA).
Clionadh Raleigh, Andrew Linke, Håvard Hegre, and Joakim Karlsen. 2010. Introducing acled: an armed conflict location and event dataset: special data feature. Journal of peace research, 47(5):651-660.

Nitin Ramrakhiyani, Swapnil Hingmire, Sangameshwar Patil, Alok Kumar, and Girish Palshikar. 2021. Extracting Events from Industrial Incident Reports . In Proceedings of the 4th Workshop on Challenges and Applications of Automated Extraction of Sociopolitical Events from Text (CASE 2021), online. Association for Computational Linguistics (ACL).

Shaina Raza. 2021. Automatic Fake News Detection in Political Platforms - A Transformer-based Approach . In Proceedings of the 4th Workshop on Challenges and Applications of Automated Extraction of Sociopolitical Events from Text (CASE 2021), online. Association for Computational Linguistics (ACL).

Francesco Re, Daniel Vegh, Dennis Atzenhofer, and Niklas Stoehr. 2021. Team "DaDeFrNi" at CASE 2021 Task 1: Document and Sentence Classification for Protest Event Detection. In Proceedings of the 4th Workshop on Challenges and Applications of Automated Extraction of Socio-political Events from Text (CASE 2021), online. Association for Computational Linguistics (ACL).

Parang Saraf and Naren Ramakrishnan. 2016. Embers autogsr: Automated coding of civil unrest events. In Proceedings of the 22nd ACM SIGKDD International Conference on Knowledge Discovery and Data Mining, KDD '16, page 599-608, New York, NY, USA. Association for Computing Machinery.

James Scharf, Arya D. McCarthy, and Giovanna Dore. 2021. Characterizing News Portrayal of Civil Unrest in Hong Kong, 1998-2020. In Proceedings of the 4th Workshop on Challenges and Applications of Automated Extraction of Socio-political Events from Text (CASE 2021), online. Association for Computational Linguistics (ACL).

Philip A. Schrodt. 2020. Keynote abstract: Current open questions for operational event data. In Proceedings of the Workshop on Automated Extraction of Socio-political Events from News 2020, page 8, Marseille, France. European Language Resources Association (ELRA).

Ralph Sundberg, Kristine Eck, and Joakim Kreutz. 2012. Introducing the ucdp non-state conflict dataset. Journal of Peace Research, 49(2):351-362.

Fiona An Ting Tan, Sujatha Das Gollapalli, and SeeKiong Ng. 2021. NUS-IDS at CASE 2021 Task 1: Improving Multilingual Event Sentence Coreference Identification With Linguistic Information. In Proceedings of the 4th Workshop on Challenges and Applications of Automated Extraction of Sociopolitical Events from Text (CASE 2021), online. Association for Computational Linguistics (ACL). 
Hristo Tanev, Jakub Piskorski, and Martin Atkinson. 2008. Real-time news event extraction for global crisis monitoring. In Natural Language and Information Systems, pages 207-218, Berlin, Heidelberg. Springer Berlin Heidelberg.

Dimitrios Tsarapatsanis and Nikolaos Aletras. 2021. On the ethical limits of natural language processing on legal text. In Proceedings of the 59th Annual Meeting of the Association for Computational Linguistics, Online. Association for Computational Linguistics.

Sander Bijl de Vroe, Liane Guillou, Miloš Stanojević, Nick McKenna, and Mark Steedman. 2021. Modality and Negation in Event Extraction. In Proceedings of the 4th Workshop on Challenges and Applications of Automated Extraction of Socio-political Events from Text (CASE 2021), online. Association for Computational Linguistics (ACL).

Wei Wang, Ryan Kennedy, David Lazer, and Naren Ramakrishnan. 2016. Growing pains for global monitoring of societal events. Science, 353(6307):15021503.

Nils B. Weidmann and Espen Geelmuyden Rød. 2019. The Internet and Political Protest in Autocracies, chapter Coding Protest Events in Autocracies. Oxford Studies in Digital Politics, Oxford.

Erdem Yörük, Ali Hürriyetoğlu, Çağrı Yoltar, and Fırat Duruşan. 2021. Random Sampling in Corpus Design: Cross-Context Generalizability in Automated Multicountry Protest Event Collection. American Behavioral Scientist, 0(0):00027642211021630.

Zhihan Zhou, Liqian Ma, and Han Liu. 2021. Trade the event: Corporate events detection for news-based event-driven trading. In Proceedings of the 59th Annual Meeting of the Association for Computational Linguistics, Online. Association for Computational Linguistics. 